\title{
Quando è che tu diventa te?
}

\author{
Raffaella Setti
}

PUBBLICATO: 20 NOVEMBER 2020

\section{Quesito:}

Sono molti i lettori che pongono domande intorno all'uso del pronome personale te: si può usare anche come soggetto? Se si, lo si può fare in qualsiasi contesto o solo in particolari condizioni? Si tratta di un fenomeno regionale o dell'italiano comune?

\section{Quando è che tu diventa te?}

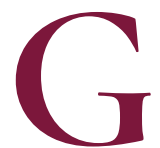

li stessi utenti, con la formulazione delle loro domande, ci guidano nell'analisi di questo fenomeno in atto nell'italiano. Con la loro competenza di parlanti nativi sono riusciti infatti a intravedere le diverse ragioni che hanno portato allo spostamento del pronome personale te dalla pressoché esclusiva funzione di oggetto (e complemento indiretto preceduto da preposizione) a quella di soggetto in particolari contesti e in determinate aree italiane (ormai decisamente estese). Un'espansione che già molti anni aveva indotto Edoardo Blasco Ferrer a questa previsione: "non è necessario essere un futurologo di professione per intuire che l'italiano del Duemila possederà saldamente la struttura io e te e forse anche l'impiego esclusivo del te come soggetto" (Io e te in "Studi linguistici italiani", XVIII I992, pp. 45-7I) e che, molto recentemente, ha fatto dire a Luca Serianni: "te ha abbastanza carte da giocare" (Il sentimento della lingua, Bologna, il Mulino 2019, p. 75). Proviamo allora a scoprire queste carte e, seguendo la traccia delle domande poste, cerchiamo di analizzare come e perché è avvenuta, e sta ancora procedendo, questa presa di campo di te a scapito di $t u$.

Partiamo dalla norma, dalla grammatica, e dalle domande in cui è posto il problema della "correttezza": "Si può usare te come soggetto? quando è corretto usare te al posto di tu?"; "Il mio amico sosteneva che te, al posto del soggetto tu, fosse un'alternativa, per cosí dire, 'standard' al soggetto tu"; "si sente continuamente in tv te invece di tu (te fai, te vai, te vieni...). È possibile che nessuno intervenga per correggere?".

Le regole che troviamo nelle grammatiche partono dallo schema tradizionale dei pronomi personali indicando, per la seconda persona singolare, le due forme, tu per il soggetto e te per gli altri complementi; questa rassicurante distinzione iniziale è seguita da alcune precisazioni che immediatamente suggeriscono quanto la "regola" sia mobile e soggetta a variazioni. In primo luogo, ci sono le eccezioni contemplate dalla stessa norma (cfr. Serianni 2000, VII, 9): paragoni di uguaglianza, "faccio come te"; espressioni esclamative, "povero te!"; quando il pronome ha funzione predicativa rispetto a un soggetto diverso, "vuole essere te in ogni cosa"; con un participio assoluto, "te compreso"; nell'uso letterario in forme latineggianti di accusativo+infinito "E se conoscerai te non essere non uomo" (L.B. Alberti, Libri della Famiglia, Libro I, 24). Già da questi casi si deduce come il sistema dei pronomi personali dell'italiano non sia cosi stabile relativamente alla distribuzione funzionale delle sue forme. Un cambiamento ormai avvenuto e acquisito anche dai grammatici è quello del sopravanzamento delle terze persone singolari e plurali lui/lei/loro soggetti rispetto ai canonici egli/ella/essi: tale spostamento ne favorisce di analoghi per altre forme, come appunto per la seconda persona $t u / t e$. E questa è una spinta forte nella direzione di una convergenza a un'unica forma, specialmente in una lingua come l'italiano in cui l'esplicitazione del pronome soggetto non è obbligatoria e, ancor più facilmente alla terza persona, in cui egli/lui (e i corrispondenti plurali 
essi/loro) possono essere sostituiti da un nome, passaggio non consentito per le prime persone. Si tratta di un altro fattore che, in questo processo già notevolmente avanzato, favorisce la forma te, presente in costrutti in cui si intende mettere in rilievo, dare incisività al soggetto, in particolare quando si trovi in una posizione sintattica non regolare, dopo il verbo ("lo dici te") o, come vocativo, fuori dal nucleo della frase ("beato te!").

Nonostante il "movimento" in atto, alcune grammatiche mantengono indicazioni nette (e non solo nei manuali scolastici, tradizionalmente più conservatori), come ad esempio quella di Aldo Gabrielli nel suo Il piacere dellitaliano (Milano, Mondadori, I999, p. 7I, il cui testo è sostanzialmente analogo a quello citato in rete e facilmente consultabile nel sito del "Corriere della Sera"):

È corretto dire, come molti dicono, hai ragione te, vieni anche te? Dubbio presto risolto: bisogna dare del $t u$, non del te, e dire hai ragione tu, vieni anche tu. La grammatica insegna che il pronome personale tu è d'obbligo come soggetto, mentre te si usa nei complementi, come quando diciamo "io (soggetto) partirò con te (complemento di compagnia)".

Una maggiore attenzione all'evoluzione in atto dimostrano Valeria Della Valle e Giuseppe Patota (Senza neanche un errore, Milano, Sperling \& Kupfer, 2006, p. Io):

\footnotetext{
Oggi c'è una forte tendenza a usare la forma te come soggetto al posto di tu. Spesso si sente dire, soprattutto al Nord: "Te canti", "Te sei simpatica", laddove la norma grammaticale ha sempre richiesto "Tu canti", "Tu sei simpatica". [...] Molti avvertiranno la nuova abitudine come una sgrammaticatura, ma questa è analoga a quella che, per il soggetto di terza persona, ha visto affermarsi la forma lui ai danni di egli. Il nostro consiglio è di evitare (ancora per qualche anno, fino a quando non si sarà completamente affermato) l'uso di te come soggetto esclusivamente nella lingua scritta di tono sorvegliato, accogliendo in questa solo il tipo "Tu vieni con noi?" "Tu non puoi venire".
}

Stefano Telve poi (Enciclopedia dellitaliano Treccani, Prontuario, 2011, p. I675, disponibile anche in rete) registra altri contesti in cui te soggetto avrebbe conquistato posizioni rispetto al tu nell'italiano contemporaneo:

a) "obbligatoriamente e in tutt'Italia, dopo le congiunzioni e e o" (ormai riconosciuta la correttezza di "io e te", si veda a tale proposito la scheda di Matilde Paoli su io e te);

b) "a livelli colloquiali, in frasi interrogative e imperative" ("te da dove vieni?", "te torna qua");

c) "quando il soggetto è dopo il verbo" ("lo dici te"), la posizione post-verbale, tipica del complemento oggetto, favorisce questo passaggio;

d) "in posizione più marcata, prima del verbo, a inizio di frase" ("te prova ad andar sotto un camion", Francesco Guccini, I fichi).

Gli ultimi tre punti presi in esame da Telve riguardano costrutti focalizzati che, dal parlato di registro informale, dove l'intonazione contribuisce in modo determinante alla marcatezza del pronome, tendono a filtrare nella scrittura o per intenti consapevoli di mimesi della lingua parlata, o per influsso del mezzo utilizzato (si pensi solo agli usi immediati e informali del linguaggio della rete). Data la natura deittica del pronome di seconda persona, quindi la necessità, all'interno dello scambio dialogico, della presenza, reale o virtuale, dell'interlocutore, queste forme compaiono in contesti in cui ci si può rivolgere a un "tu" ascoltatore o lettore, in cui - come ha ben sintetizzato Enrico Testa - si ha la «messa in scena dell'enunciazione "parlata" e della lingua in azione dei suoi protagonisti» con la presenza frequente di moduli di forte oralità (E. Testa, Lo stile semplice, Torino, Einaudi, p.r38). Questo comporta che, nella scrittura, il luogo naturale per queste forme sia il discorso diretto (o la sua simulazione); inoltre, come accennato, il te soggetto è caratteristico di registri colloquiali e 
informali: condizioni decisamente difficili da isolare in una ricerca su banche dati testuali o addirittura in rete, anche senza considerare la difficoltà di distinguere i te soggetto da tutti gli altri ruoli che il pronome può ricoprire. Valutare quantitativamente l'incidenza di quest'uso in rete risulta dunque pressoché impossibile, troppi i fattori di "rumore". Possiamo provare a farci un'idea molto approssimativa attraverso coppie di stringhe in cui si alternano tu e te.

Sulle pagine in italiano di Google (3/6/2020) abbiamo messo a confronto le seguenti coppie ottenendo questi risultati in termini di occorrenze:

I. "ma tu sei di": I07.00o / "ma te sei di": I98.00o (in contesti del tipo: "Ma te sei di NapoliNapoli?", "Ma te sei di Indicatore? Io di Ponticino" "ma te sei di natura molto scettico", "ma te sei di fuori come i terrazzi", "ma te sei di parte", "ma te sei di sinistra");

2. "ma tu hai": 892.000 / "ma te hai": 207.000;

3. "solo perché sei tu": 97.Ioo / "solo perché sei te": I0.700;

4. "hai ragione tu": I82.000 / "hai ragione te": 27.300;

5. "vieni anche tu": I78.000 / "vieni anche te": II.500.

L'impressione che possiamo trarre da questi dati è che, almeno per le stringhe indagate, il tu tiene, salvo un lieve cedimento nel primo caso. Ripetendo le stesse ricerche su Google Books Ngram Viewer (che permette il confronto tra le stringhe nel corpus dei testi in italiano presenti in Google dal i8oo al 20I2) notiamo che solo l'ultima stringa restituisce un grafico di confronto (sotto), mentre tutte le altre danno valori non rilevabili per le stringhe con il pronome te.

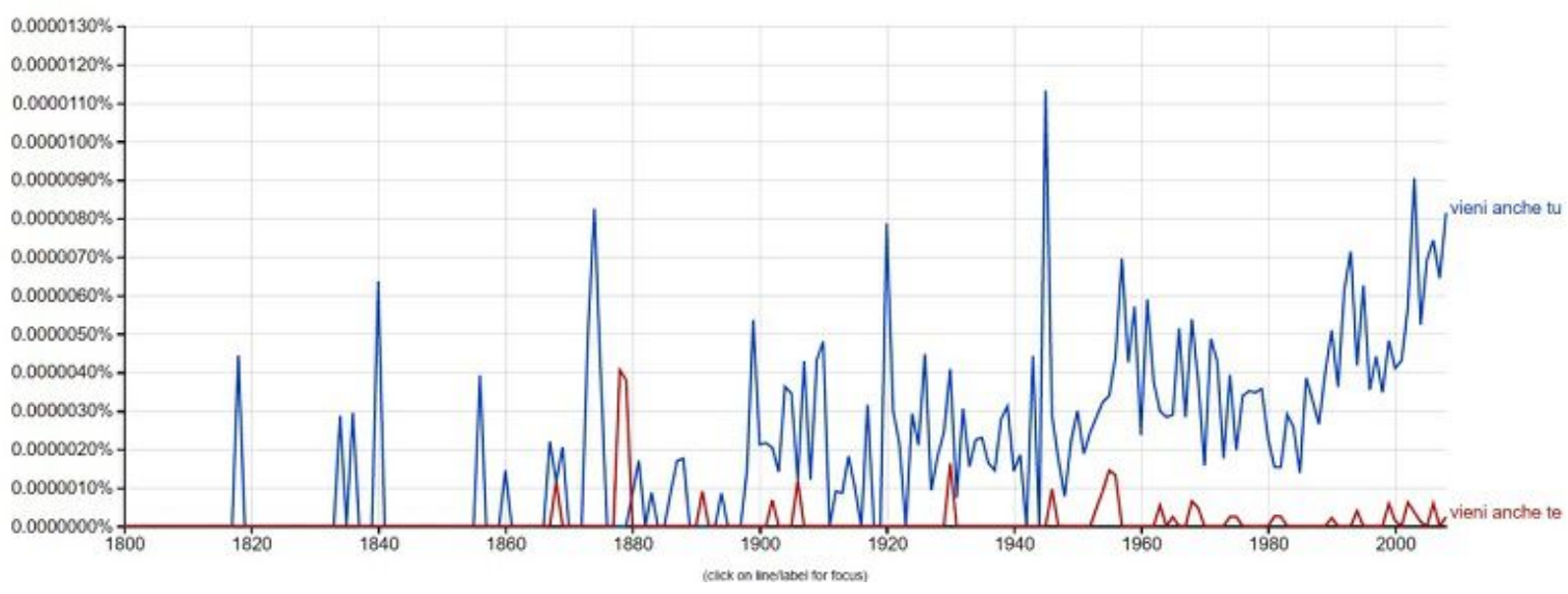

Il secondo aspetto messo in rilievo da alcune domande riguarda le diverse scelte dei parlanti in relazione alla provenienza regionale: "Da quando mi sono trasferito al nord Italia, non sento quasi mai il pronome personale TU usato come soggetto. Al nord generalmente (quasi sempre) si usa il complemento TE anche come soggetto". Tali osservazioni aprono una questione sempre presente quando si vada a indagare fenomeni di variazione negli usi: la distribuzione regionale delle diverse forme dovuta ai sostrati dialettali. Anche in questo caso si è cercato di individuare una zona di origine e irraggiamento del fenomeno, documentato senz'altro nella parlata familiare toscana e dato da Rohlfs (Cfr. Rohlfs I968, § 435 e 453) come "abbastanza frequente" nel vernacolo toscano che prevede anche la reduplicazione del pronome (del tipo "te tu dici"): il pronome soggetto te, tipico dunque della Toscana, si sarebbe poi esteso alle zone confinanti, nel Lazio con Roma, dove il fenomeno si diffonde in epoca postunitaria, portato dai "buzzurri" toscani e piemontesi arrivati nella nuova capitale, e poi nelle Marche e, a nord a Bologna. Nel nord Italia, il sostrato dialettale ha influito su questo cambiamento dell'italiano: infatti, nei dialetti settentrionali, si è verificato il passaggio da tu (in realtà 
ti) a te e tale evoluzione ha interessato anche la prima persona (da io, anzi eo a mi), anche se non ha prodotto poi me soggetto in italiano (a parte nelle espressioni esclamative del tipo povero me!). Ma l'influsso toscano e la spinta dei dialetti settentrionali non sembrano sufficienti a spiegare una diffusione cosi ampia che ormai comprende buona parte del settentrione e tutto il centro Italia, comprese le varietà romana e napoletana. A partire dalle considerazioni già contenute nell'articolo di Blasco Ferrer (citato all'inizio), le più recenti analisi dell'italiano contemporaneo sono concordi nell'individuare cause autonome per spiegare la diffusione del fenomeno nelle diverse varietà. In particolare, come sintetizzato da Lorenzetti (L'italiano contemporaneo, Roma, Carocci, 2002) e ripreso da Federica Da Milano (nel suo articolo sui Pronomi personali nell'Enciclopedia dell'Italiano Treccani):

\footnotetext{
Negli italiani settentrionali e nell'italiano toscano essa dipende soprattutto dai dialetti sottostanti, che esprimono obbligatoriamente il soggetto pronominale e usano frequentemente, seppure in modi diversi, un te soggetto, sia da solo sia in forme reduplicate (bolognese te $t$ port «tu porti»). Ma il te soggetto è diffuso e vitale anche in varietà regionali che, come quella romana e quella napoletana, non hanno pronomi soggetto obbligatori. Qui il pronome te come soggetto si deve a due fattori convergenti: uno, sociolinguistico, è l'influsso dell'italiano settentrionale, varietà trainante a partire dagli anni Settanta; il secondo, grammaticale, è l'indebolirsi della distinzione tra una forma soggetto e una forma complemento oggetto.
}

In questa direzione si hanno conferme anche dal LIP il corpus dell'italiano parlato che ci restituisce esempi di te soggetto in parlanti fiorentini ("da quando te sei unoccupante"; "allora Barbara te sei l'ultima"; "ma te sei un uomo"; "ma te sei matto") e romani ("guarda che te sei mancato all'ora mia"). Per la stessa sequenza te sei (non affidabili risultati sulla ricerca generica su te) ci sono 2I occorrenze anche nel LIT, Lessico Italiano Televisivo, mentre soltanto 5 nel LIR, Lessico Italiano Radiofonico; molto inferiori i numeri relativi alla sequenza te hai che si riducono a II nel LIT e a 2 soltanto nel LIR.

Accanto all'influsso dei dialetti sottostanti sulle varietà regionali si conferma quindi la pressione delle ragioni strutturali del sistema pronominale dell'italiano contemporaneo ricco, nel suo insieme, di fenomeni innovativi: l'impiego di te soggetto è stato notato nei testi delle canzoni, dal successo di Nek Laura non c'é nel passaggio ci sei te, al più recente te stai buono, te che dici? in I tuoi particolari di Ultimo (cfr. Paolo D’Achille, L'italiano contemporaneo, Bologna, il Mulino, 2019, p. II4), mentre sembra confermare la tenuta del tu nelle aree del sud Italia l'uso che ne fa Caparezza nei testi delle sue canzoni (un esempio per tutti il titolo Avrai ragione tu, per altri esempi si rimanda ad Annarita Miglietta, Sulla lingua del rap italiano, Firenze, Cesati, 2019, pp. 69-7I). Anche Fabio Rossi, pur manifestando il suo attaccamento al tu soggetto (dove ancora è vitale), individua proprio nei mutamenti interni al sistema pronominale la motivazione più forte che induce a ritenere molto probabile una definitiva sostituzione a favore di te (le "carte da giocare" di Serianni) e cosi si rivolge al suo interlocutore (si cita da una sua risposta pubblicata su DICO, Dubbi sull'Italiano Consulenza Online):

E allora, al momento abbiamo cambiato la grammatica accogliendo hii e lei soggetto, ma forse non è ancora il momento di farlo per te soggetto, dato che un numero molto elevato di parlanti e scriventi, come Lei e come chi le scrive, ancora non sente naturale il te al posto di tu e gran parte degli italiani colti a Sud di Roma la pensa come noi due, credo. Pertanto, in barba allo strapotere romano-milanese, teniamoci ancora un po' il nostro tu, senza crociate e pronti a cedere quando nessun altro, forse, ci farà più una domanda (bella) come la sua.

Un concorso di fattori dunque ha determinato il diffondersi e l'affermarsi, in molte varietà parlate dell'italiano contemporaneo, della forma te con funzione di soggetto al posto di tu; dall'oralità il tratto sta filtrando anche nell'interazione scritta nelle sue modalità più disinvolte e colloquiali (in particolare "digitate"); la norma e l'insegnamento scolastico propongono in sostanza, con più o meno segnalazioni 
rispetto agli usi divergenti, la descrizione canonica con le due forme funzionalmente distinte. Continuiamo a prevedere che l'esito sarà analogo a quello avuto da hi/lei/loro rispetto a egli/ella/essi, non prima però che te soggetto si diffonda tra i parlanti di quelle aree in cui ancora è avvertito come un corpo estraneo e filtri in maniera estesa e indolore anche nella scrittura di registro medio.

\section{Cita come:}

Raffaella Setti, Quando è che tu diventa te?, "Italiano digitale", 2020, XV, 2020/4 (ottobre-dicembre) DOI: $10.35948 / 2532-9006 / 2020.5425$

\section{Copyright 2020 Accademia della Crusca}

Pubblicato con licenza creative commons CC BY-NC-ND 